\title{
Gene discovery for the bark beetle-vectored fungal tree pathogen Grosmannia clavigera
}

\author{
Uljana Hesse-Orce ${ }^{1}$, Scott DiGuistini ${ }^{1}$, Christopher I Keeling ${ }^{2}$, Ye Wang ${ }^{1}$, Maria Li ${ }^{2}$, Hannah Henderson ${ }^{2}$, \\ T Roderick Docking ${ }^{3}$, Nancy Y Liao ${ }^{3}$, Gordon Robertson ${ }^{3}$, Robert A Holt ${ }^{3}$, Steven JM Jones ${ }^{3}$, Jörg Bohlmann², \\ Colette Breuil $^{1 *}$
}

\begin{abstract}
Background: Grosmannia clavigera is a bark beetle-vectored fungal pathogen of pines that causes wood discoloration and may kill trees by disrupting nutrient and water transport. Trees respond to attacks from beetles and associated fungi by releasing terpenoid and phenolic defense compounds. It is unclear which genes are important for G. clavigera's ability to overcome antifungal pine terpenoids and phenolics.

Results: We constructed seven cDNA libraries from eight G. clavigera isolates grown under various culture conditions, and Sanger sequenced the $5^{\prime}$ and $3^{\prime}$ ends of 25,000 cDNA clones, resulting in 44,288 high quality ESTs. The assembled dataset of unique transcripts (unigenes) consists of 6,265 contigs and 2,459 singletons that mapped to 6,467 locations on the G. clavigera reference genome, representing 70\% of the predicted G. clavigera genes. Although only $54 \%$ of the unigenes matched characterized proteins at the NCBI database, this dataset extensively covers major metabolic pathways, cellular processes, and genes necessary for response to environmental stimuli and genetic information processing. Furthermore, we identified genes expressed in spores prior to germination, and genes involved in response to treatment with lodgepole pine phloem extract (LPPE).

Conclusions: We provide a comprehensively annotated EST dataset for G. clavigera that represents a rich resource for gene characterization in this and other ophiostomatoid fungi. Genes expressed in response to LPPE treatment are indicative of fungal oxidative stress response. We identified two clusters of potentially functionally related genes responsive to LPPE treatment. Furthermore, we report a simple method for identifying contig misassemblies in de novo assembled EST collections caused by gene overlap on the genome.
\end{abstract}

\section{Background}

The ophiostomatoid fungus Grosmannia clavigera (Robinson-Jeffrey and Davidson) is a fungal pathogen that discolours wood, and kills pine host trees by disrupting the flow of nutrients and water in phloem and sapwood [1,2]. In its ecosystem, G. clavigera is vectored between hosts by the mountain pine beetle (MPB, Dendroctonus ponderosae). This pathogen can kill lodgepole pine (Pinus contorta) when manually inoculated under the bark at a high enough concentration $[3,4]$. Like many bark beetle associated fungi, G. clavigera produces slimy spores that stick to the exoskeleton of the insects, but the fungus is also present in the beetle mycangia

\footnotetext{
* Correspondence: colette.breuil@ubc.ca

'Department of Wood Science, University of British Columbia, Vancouver, Canada

Full list of author information is available at the end of the article
}

and alimentary canal [5]. During tree colonization, the beetles spread fungal spores throughout their galleries below the bark. The spores germinate and fungal hyphae colonize the phloem and sapwood of the tree. Fungi benefit the beetles by improving the host environment for the beetle progeny, and serving as food for the larvae and the teneral adult beetles [6]. In addition, G. clavigera may counteract tree defenses that are activated during bark beetle attacks.

Conifer trees respond to beetle attacks or fungal inoculation by releasing resin from pre-formed and inducible traumatic resin ducts, inducing the synthesis of phenolic compounds in phloem parenchyma cells, and forming a wound periderm tissue [7]. The main constituents of conifer resin are terpenoids, many of which have insecticidal and fungicidal properties $[8,9]$. Ophiostomatoid fungi can decrease the concentration of
C Biomed Central

(c) 2010 Hesse-Orce et al; licensee BioMed Central Ltd. This is an Open Access article distributed under the terms of the Creative Commons Attribution License (http://creativecommons.org/licenses/by/2.0), which permits unrestricted use, distribution, and reproduction in any medium, provided the original work is properly cited. 
terpenoids when inoculated on sapwood [10-12]. However, the molecular and biochemical mechanisms involved in these processes are unknown. Previously, we analyzed a small set of expressed sequence tags (ESTs) from G. clavigera and found putative gene candidates that may be involved in terpenoid detoxification [13]. Lodgepole pine also constitutively produces a variety of phenolic compounds, including flavonoids and tannins [14-17]. Many of these chemicals inhibit the growth of fungal pathogens [18]. To detoxify phenolic plant defense compounds, fungi produce a variety of enzymes such as phenol oxidases that polymerize phenolics [19], peroxidases that degrade polymeric phenolic structures [20], and glucosidases and glucuronidases that are involved in metabolism of phenolic glycosides [21,22]. As well, fungi may release extracellular proteins that bind to toxic phenolics preventing their interaction with the fungal cell wall [23]. Whether G. clavigera encodes and expresses genes necessary for detoxification of phenolic compounds has not been determined.

The recently published genome of $G$. clavigera is the first reference genome for an insect-vectored fungal tree pathogen [24]. The genome sequence provides a fundamental resource for identifying fungal genes important for symbiotic interactions with insect and tree hosts. ESTs support gene discovery and gene structure annotation. To cover a broad spectrum of expressed genes, we extended the previous collection of 5,950 ESTs [13], and sequenced the 5' and 3' ends of 25,000 cDNA clones from normalized and non-normalized cDNA libraries of eight G. clavigera isolates grown in various conditions. We conducted a de novo EST assembly and used the resulting set of unique transcripts (unigenes) to verify the assembly of the G. clavigera genome [24]. As well, we used the genome sequence to address two problems associated with de novo CAP3 EST assembly: 1) incomplete assembly of ESTs from multiple transcripts of a given gene into a single contig due to incomplete splicing, splice variants, incomplete read overlap, and sequencing errors; and 2) incorrect assembly of ESTs from transcripts of different genes as a result of sequence similarity.

Here we describe the G. clavigera unigene dataset, and discuss fungal genes upregulated in two biological states that are important for G. calvigera-MPB-pine interactions: the vectored non-germinating asexual spores, and mycelia treated with lodgepole pine phloem extract (LPPE), which contains tree defense chemicals. Furthermore, we show that overlap of neighboring genes on the genome was the major source for contig misassemblies in this de novo EST assembly, and describe a simple method for identifying such cases even in absence of a sequenced genome.

\section{Results}

1 EST analysis

To identify a broad spectrum of G. clavigera genes necessary for fungal growth we grew eight isolates (Table 1) on five media, and for one medium we treated the mycelia with LPPE (Table 2). We also harvested spores from the reference isolate SLKW1407 that we had used to sequence the G. clavigera genome [24]. To maximize sequencing efficiency we pooled the media and LPPE treatments, generating three isolate/treatment combinations with the reference isolate, and one isolate/ treatment combination that contained the seven closely related G. clavigera isolates (Table 3). Furthermore, we prepared normalized cDNA samples for three isolate/ treatment combinations. We then constructed seven unidirectional, full-length enriched cDNA libraries and sequenced a total of 25,000 cDNA clones from both $5^{\prime}$ and 3' ends (Table 3). After trimming vector and low quality sequences the average PHRED 20 read length was 693 bp. This resulted in 44,288 high quality ESTs (NCBI dbEST GT571598-GT615878). After adding 5,950 quality-filtered ESTs from previous analyses with the reference isolate [13], we reverse-complemented 3' reads, removed polyA tails, and discarded sequences with long mononucleotide stretches. The resulting dataset contained 50,167 high quality ESTs.

Table 1 G. clavigera strains isolated from Pinus species used for cDNA library construction

\begin{tabular}{|c|c|c|c|c|c|c|}
\hline ID & Isolate & ATCC/UAMH accession & Isolation origin & Host & Isolated from & Year \\
\hline 1 & SLKW1407 & UAMH11150 & BC/Kamloops & P. contorta & Gallery & 2003 \\
\hline 2 & ATCC18086 & ATCC18086 & BC/Cache Creek & P. ponderosa & Sapwood & 1965 \\
\hline 3 & $200-1-14$ & UAMH11151 & BC/Kamloops & P. contorta & Sexual spore & 2004 \\
\hline 4 & DPLKGT1B & UAMH11152 & BC/Kelowna & P. contorta & MPB body & 2007 \\
\hline 5 & H55 & UAMH11153 & BC/Houston & P. contorta & MPB body & 2003 \\
\hline 6 & B5 & UAMH11154 & Alberta/Banff & P. contorta & MPB body & 2003 \\
\hline 7 & B10 & UAMH11155 & Alberta/Banff & P. contorta & MPB body & 2003 \\
\hline 8 & DPCHMC3 & * & Alberta/Cypress Hills & P. contorta & MPB mycangia & 2007 \\
\hline
\end{tabular}

*Breuil culture collection, Dept. of Wood Science, University of British Columbia, Vancouver, Canada 
Table 2 Media and treatment for growing G. clavigera mycelium for cDNA library construction

\begin{tabular}{ll}
\hline Media & composition \\
\hline wood & $10 \mathrm{~g} /$ plate lodgepole pine sawdust \\
starch & $0.17 \%$ YNB, 0.1\% PHP, 0.3\% asparagine, 1\% starch \\
organic & $0.17 \%$ YNB, 0.1\% PHP, 0.3\% asparagine, 1\% maltose \\
nitrogen & \\
inorganic & $0.17 \%$ YNB, 0.1\% PHP, 0.3\% NaNO3, 1\% maltose \\
nitrogen & \\
olive oil & $\begin{array}{l}0.17 \% \text { YNB, 0.1\% PHP, 0.3\% asparagine, 1\% ( } / \mathrm{V} \text { ) olive } \\
\text { oil emulsified in 0.5\% tergitol (olive oil and tergitol } \\
\text { were mixed and autoclaved separately before being } \\
\text { added to the media) }\end{array}$ \\
\hline $\begin{array}{l}\text { LPPE } \\
\text { treatment }\end{array}$ & $\begin{array}{l}\text { Cultures were grown on organic nitrogen medium for } \\
\text { hend then sprayed with LPPE }\end{array}$
\end{tabular}

All media were solidified with $1.5 \%$ granulated agar. LPPE = lodgepole pine methanol extract, YNB = yeast nitrogen base, PHP = potassium hydrogen phthalate

Unigene assembly and unigene-locations on the genome We assembled the 50,167 ESTs into unigenes and determined their locations on the G. clavigera genome. Assembly resulted in 8,724 unigenes $(2,459$ singletons and 6,265 contigs) that joined $91 \%$ of the 21,391 5'-3' read pairs. Most of the unigenes (97\%) mapped to the genome at high stringency ( $>80 \%$ sequence similarity; $>80 \%$ unigene alignment length), while 219 unigenes mapped with a quality below this threshold, and 48 unigenes did not align to the genome. We evaluated the contig assembly using two methods. First, we aligned the unassembled reads to the genome and tested whether all reads from a contig would map to the same genome location as the contig. For 74 contigs there was disagreement. Manual inspection showed that 28 were correctly assembled and could be matched to an NCBI protein sequence, leaving $46(0.5 \%)$ potentially misassembled unigenes. Second, we searched for contigs containing forward and reverse reads (FR contigs) despite unidirectional orientation of the assembled reads. We identified 821 such contigs, and in most cases found indications for transcript overlap between neighboring genes encoded on opposite genome strands. For all but 21 (2.6\% of 821$)$ FR contigs, the unassembled reads mapped to the same genome location as their respective contig aligning, however, to both strands of the genome. We manually inspected 50 FR contigs and found that for 35 of them the reverse assembled reads belonged to an adjacent gene model encoded on the opposite strand. For the remaining 15 FR contigs all reads mapped to the same region on the genome, but we found no evidence for a neighboring gene.

We generated 6,467 unigene-locations (ULs) on the G. clavigera reference genome based on strand-specific unigene overlap and linking information from unassembled 5'-3' read pairs (Additional file 1: Table S1). Of these, 812 ULs (13\%) overlapped with another UL encoded on the opposite strand of the genome, with a 260 bp median overlap length. However, FR contig analysis suggested that UL overlap occurs more frequently. For the 814 ULs with FR contigs, UL analysis identified 170 overlapping ULs (21\%). Manual inspection of genome locations with FR contigs indicated this fraction to be $\sim 70 \%$ (i.e. $\sim 570$ ULs). This suggested that overall $\sim 1212$ G. clavigera ULs overlapped.

\section{Genes represented in the unigene dataset}

We predicted ORFs for all but five of the 8,724 unigenes; 1,584 of these unigenes (18\%) were full length, 4,806 were truncated at the 5 ' end, and 771 were truncated at the 3' end. Seventy-three percent of the unigenes were similar to NCBI protein sequences (min evalue $\left.1.0 \times 10^{-4}\right)$. For these unigenes ORF predictions were based on the best BLAST match, and we found that for 1,115 of them the best predicted ORF was not the longest ORF. We also noted that only $8 \%$ of the correctly assembled unigenes had a significant protein

Table 3 cDNA libraries from four G. clavigera isolate-treatment combinations and numbers of high quality ESTs derived from each library.

\begin{tabular}{lclcccc}
\hline Library & Iso-lates & Media/treatment combinations & Norma-lized & Primary titre (cfu/ml) & ESTs sequenced & ESTs HQ (\%) \\
\hline OCL01 & 1 & W:S:ON:IN:OO & No & $2.1 \times 10^{6}$ & 6,144 & $5,272(86)$ \\
OCL02 & 1 & W:S:ON:IN:OO & Yes & $2.1 \times 10^{5}$ & 15,360 & $14,075(92)$ \\
\hline OCL03 & 1 & ON+LPPE & No & $4.0 \times 10^{5}$ & 6,144 & $5,618(91)$ \\
OCL04 & 1 & ON+LPPE & Yes & $2.5 \times 10^{5}$ & 9,216 & $7,794(85)$ \\
\hline OCL05 & $2-8$ & W:S:ON:IN:OO:ON+LPPE & No & $2.0 \times 10^{6}$ & 3,072 & $2,916(95)$ \\
OCL06 & $2-8$ & W:S:ON:IN:OO:ON+LPPE & Yes & $9.0 \times 10^{4}$ & 3,072 & $2,819(92)$ \\
\hline OCL08 & 1 & Sp & No & $1.3 \times 10^{6}$ & 6,912 & $5,794(84)$ \\
\hline
\end{tabular}

For the libraries OCL01-OCL06, mycelial cultures from each of the eight isolates were grown on five different media, and for one medium the mycelia were treated with LPPE $(\mathrm{W}=$ wood, $\mathrm{S}=$ starch, $\mathrm{ON}=$ organic nitrogen, $\mathrm{IN}=$ inorganic nitrogen, $\mathrm{OO}=$ olive oil, $\mathrm{LPPE}=$ treatment with lodgepole pine methanol extract). After extracting total RNA separately from each of the 48 isolate/media/LPPE-treatment variants and from the spore sample (Sp), equal amounts of total RNA were pooled to obtain four isolate/treatment combinations. From these pooled samples we purified poly(A+) mRNA and generated cDNA. All three nonspore cDNA samples were divided into two fractions, one of which was normalized. The resulting seven cDNA samples were used for library construction. HQ = high quality. 
match on the reverse strand, while $56 \%$ of the FR contigs had a significant hit on the minus frame. Using InterProScan and KAAS, we assigned InterPro IDs, Gene Ontology terms, and K-numbers with corresponding BRITE classifications to 3,730 (43\%), 2,504 (29\%) and 1,530 (18\%) unigenes, respectively. The K-number annotated unigenes belonged to 1,439 ULs.

Hierarchical classification of the unigenes using KEGG-BRITE allowed mapping ULs to pathways and infer higher-order functions (Figure 1, Additional file 1: Table S1). Nearly half of the 1,439 ULs that were annotated with K-numbers encoded proteins from metabolic pathways, including amino acid metabolism, carbohydrate metabolism, energy metabolism, as well as lipid and nucleotide metabolism. Proteins encoded by 575 ULs are potentially involved in genetic information processing (i.e. transcription, translation, replication and DNA repair). Furthermore, we verified that this G. clavigera unigene collection covers essential metabolic pathways. Using reciprocal BLAST analysis we identified all genes of the ergosterol pathway, all but one of the genes of the citrate and pentose phosphate cycles, and 59 of 95 genes necessary for primary amino acid biosynthesis.

\section{Differential gene expression}

We characterized gene expression in the G. clavigera reference isolate (SLKW1407) by assessing EST frequencies in the non-normalized cDNA libraries from mycelial culture (OCLO1), spores (OCL08), and cultures exposed to LPPE treatment (OCL03). Transcripts for the majority of ULs $(1699 ; 65 \%)$ appeared to be library specific, 614 ULs (24\%) contained transcripts from two libraries, and only 288 ULs (11\%) contained reads from all three libraries (Figure 2). To identify genes associated with processes that were overrepresented under any of the three conditions tested, we analyzed the set of library-specific ULs that were annotated with K-numbers and assigned to KEGG Pathways (Figure 3). Table 4 shows selected pathways for which numbers of specifically expressed genes differed between the three cDNA libraries. Among the ULs expressed only in the mycelial culture library we found twice as many carbohydrate and amino acid metabolism genes than in the other libraries. Spore-library specific ULs encoded a higher variety of proteins necessary for oxidative phosphorylation, nucleotide metabolism, and translation. In the LPPE library, genes for signal transduction and $\mathrm{N}$ glycan biosynthesis were specifically expressed. Below we describe ULs that were identified as differentially expressed by reciprocal comparison of read frequencies of the three non-normalized cDNA libraries.

\section{Spores (OCL08) vs mycelial culture (OCL01)}

Of the 2,039 ULs with reads from the libraries OCL01 and/or OCL08, 66 ULs had significantly ( $p<0.05$ ) higher read frequencies in the spore than in the mycelial library (Additional file 1: Table S1). Of these, 11 ULs (355, 444, 2425, 2447, 2745, 3423, 3838, 4185, 4783,

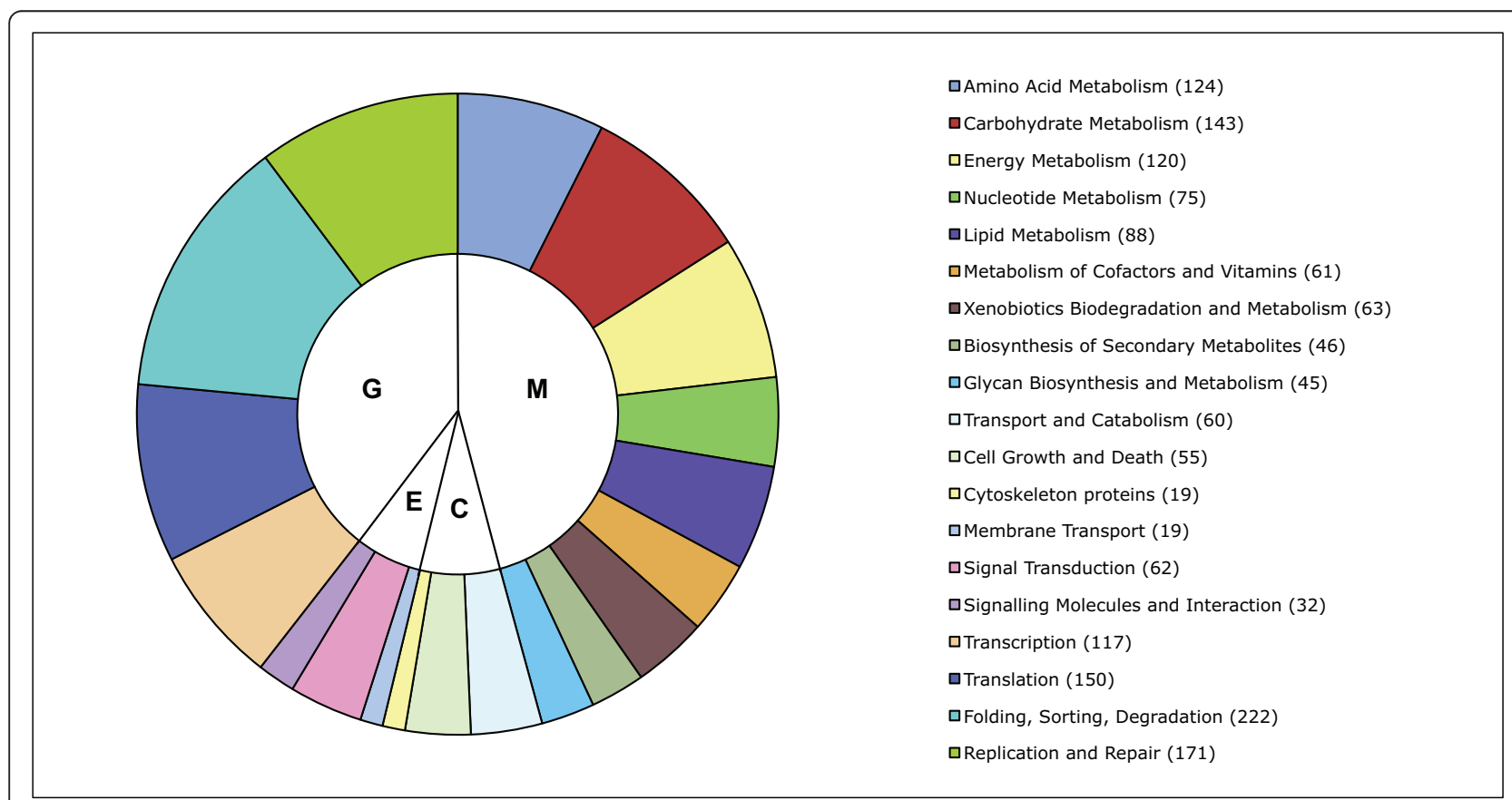

Figure 1 Numbers of unigene-locations involved in metabolic pathways (M), cellular processes (C), environmental information processing (E), and genetic information processing (G) based on KEGG-BRITE annotations. 


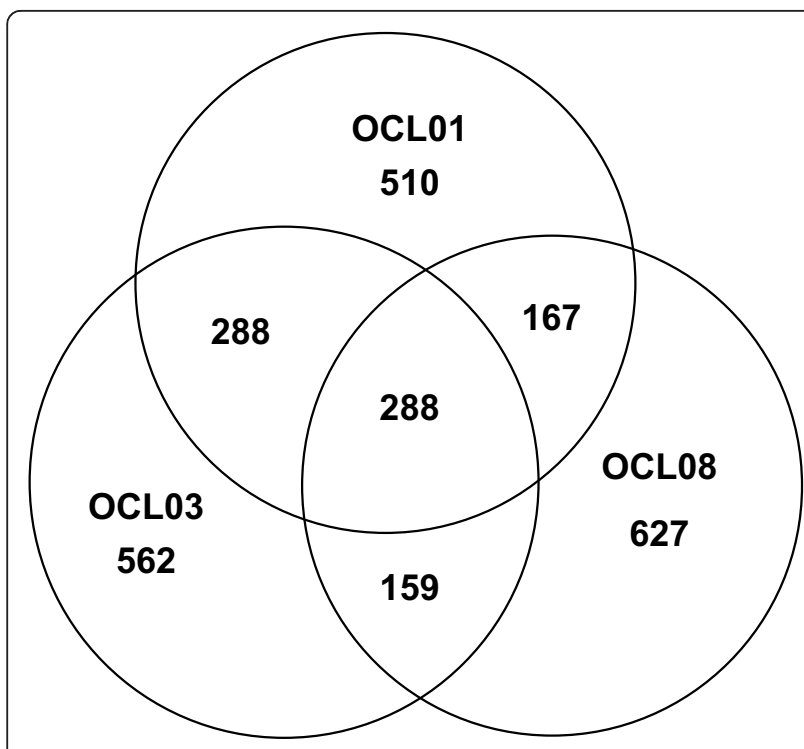

Figure 2 Numbers of unique and shared transcripts in the nonnormalized mycelial culture (OCL01), LPPE (OCL03), and spore (OCL08) CDNA libraries of G. clavigera.

$5218,5775)$ encoded heat shock proteins (HSPs) and other protein chaperones/folding catalysts (e.g. cyclophillin D). Other ULs that were overexpressed in spores matched proteins involved in energy metabolism and
ATP-dependent cellular processes: $\mathrm{Ca}^{2+}$ transporting ATPase (UL 904), F-type H+-transporting ATPase subunit epsilon (UL 2399), arsenite-transporting ATPase (UL 4670), ATP-dependent Clp protease (UL 5775). For 73 ULs read frequencies were significantly higher in the mycelial than in the spore libraries (Additional file 1: Table S1). A high fraction of ULs that were overexpressed in mycelial culture encoded proteins important for carbohydrate and amino acid metabolism: four glycolytic enzymes (ULs 2601, 4586, 6279, 6391), a glucose transporter (UL 5108), a subtilase (UL 4937), and proteins involved in methionine (ULs 1740,6245$)$ and melanin (UL 4533) biosynthesis.

LPPE treatment (OCL03) vs mycelial culture (OCL01)

We identified eight ULs $(85,588,1906,2850,3233$, $3525,4003,4803)$ that were significantly $(\mathrm{p}<0.05)$ overexpressed in the LPPE treated culture library (Additional file 1: Table S1). We analyzed the genomic neighborhoods of these eight ULs and found that two ULs (annotated as a steroid monoxygenase and a cupin domain protein) co-localized with other ULs that were expressed only in the LPPE treated libraries, and so may be functionally related. The first cluster (Table 5) contained the steroid monoxygenase and ULs encoding a beta-lactamase (whose ESTs originated from the

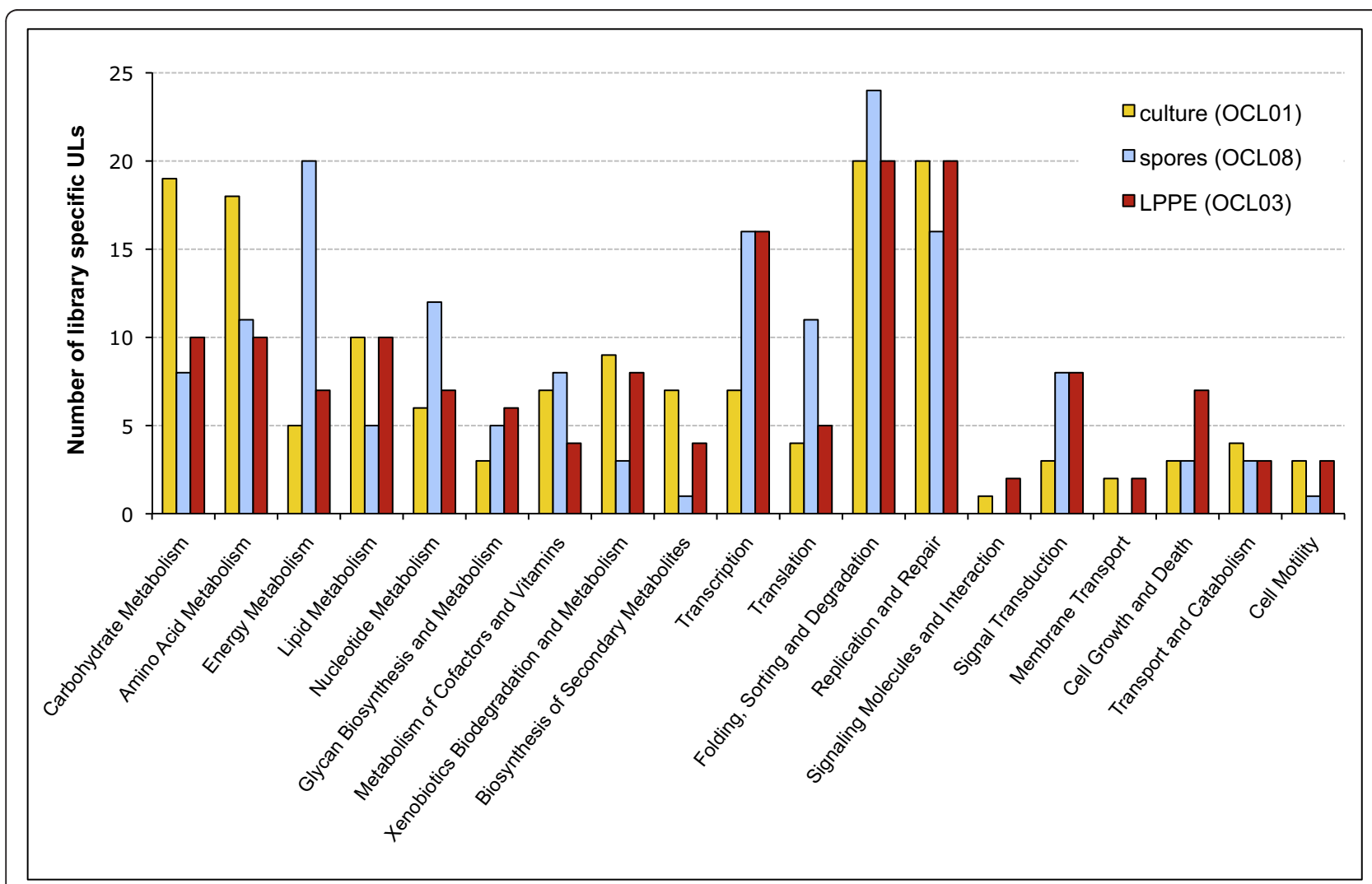

Figure 3 Library specific unigene-locations (ULs) from the non-normalized mycelial culture (OCL01), LPPE (OCL03), and spore (OCL08) CDNA libraries classified based on KEGG-BRITE annotations ( $n_{\mathrm{OCL} 01}=141, \mathrm{n}_{\mathrm{OCLO3}}=126, \mathrm{n}_{\mathrm{OCL} 08}=156$ ) 
Table 4 Numbers of genes in selected KEGG pathways matched by ULs specific to cDNA libraries OCL01 (mycelial culture), OCL03 (LPPE), and OCL08 (spores)

\begin{tabular}{|c|c|c|c|}
\hline Pathway & OCL01 & OCL03 & OCL08 \\
\hline Oxidative phosphorylation & 1 & 3 & 14 \\
\hline Amino sugar and nucleotide sugar metabolism & 6 & 2 & 1 \\
\hline Starch and sucrose metabolism & 5 & 1 & 0 \\
\hline Phenylalanine, tyrosine and tryptophan biosynthesis & 5 & 1 & 1 \\
\hline N-Glycan biosynthesis & 0 & 3 & 2 \\
\hline High-mannose type N-glycan biosynthesis & 2 & 0 & 1 \\
\hline O-Mannosyl glycan biosynthesis & 0 & 1 & 1 \\
\hline Biosynthesis of terpenoids and steroids & 3 & 6 & 0 \\
\hline Pyrimidine metabolism & 3 & 6 & 8 \\
\hline Purine metabolism & 4 & 4 & 11 \\
\hline RNA polymerase & 0 & 2 & 7 \\
\hline Spliceosome & 3 & 9 & 5 \\
\hline Aminoacyl-tRNA biosynthesis & 2 & 1 & 6 \\
\hline MAPK signaling pathway - yeast & 0 & 4 & 1 \\
\hline Cell cycle - yeast & 1 & 6 & 2 \\
\hline
\end{tabular}

normalized LPPE library), an MSF transporter, a cytochrome P450, and a P450 reductase. RT-PCR confirmed that transcript abundance for all five of these clustered ULs were increased in response to LPPE treatment at the $36 \mathrm{~h}$ time point (Figure 4). Reciprocal BLAST searches indicated that this cluster is not conserved in Aspergillus spp., Fusarium spp., Magnaporthe grisea, and Neurospora crassa. However, in Aspergillus nidulans, the putative orthologues of the cytochrome P450 (AN5837) and the P450 reductase (AN5838) are located next to each other. The second cluster (Table 6) contained the cupin domain protein and ULs for an S15 family peptidase, an unknown protein, an aromatic ringopening dioxygenase, a PutA family dehydrogenase, and a short chain dehydrogenase. Three ULs of this cluster were specific to the LPPE treatment libraries. This cluster was not conserved in the above fungal species. For 11 ULs $(602,769,2601,3744,3874,4487,4533,5218$, $5833,6220,6391)$, read frequencies were significantly (p $<0.05$ ) lower in the LPPE library than in the mycelial library (Additional file 1: Table S1). Eight of these, including the ULs for trehalase, scytalone dehydratase 1, and HSP90 were also downregulated in the spore library. Analysis of the genomic regions flanking these ULs did not indicate clustering of functionally related genes.

\section{Discussion and Conclusions}

ESTs can be assembled de novo, or, when a high-quality reference genome sequence is available, by mapping sequence reads to the genome and assembling them by genome location. We conducted a de novo EST assembly to assess the quality of the G. clavigera genome sequence [24]. For this, we applied CAP3, a widely used EST assembly program that is a component of recent assembly pipelines $[25,26]$. Of the 8,457 unigenes, $97 \%$ mapped to the genome at high stringency ( $>80 \%$ sequence similarity; $>80 \%$ unigene alignment length). Only $0.5 \%$ of the unigenes contained reads from distant genome locations, indicating either unigene or genome misassemblies. These results show that both the assembled genome sequence and the unigene assembly were of high quality. The unigene collection derived from 50,167 high quality ESTs allowed identification of

Table 5 Unigene-locations of cluster 1, which is potentially involved in response to lodgepole pine phloem extract

\begin{tabular}{|c|c|c|c|}
\hline Unigene-location & Best annotated protein match from the NCBI nonredundant protein database & e-value & p-value* \\
\hline 3230 & MFS sugar transporter [Aspergillus flavus] & $4 \mathrm{E}-92$ & ns \\
\hline 3231 & related to ARCA protein [Neurospora crassa] & $4 \mathrm{E}-38$ & ns \\
\hline Gc_00052 & benzoate 4 monooxygenase cytochrome P450 [Neosatoria fisherii] & $1 \mathrm{E}-165$ & ns \\
\hline Gc_00102 & NADPH-cytochrome P450 reductase (CprA) [Aspergillus fumigatus] & $4 \mathrm{E}-143$ & ns \\
\hline 3232 & small s protein [Podospora anserina] & $7 \mathrm{E}-42$ & ns \\
\hline 3233 & steroid monooxygenase [Aspergillus flavus] & $1 E-104$ & 0.02 \\
\hline 3234 & beta-lactamase family protein [Pyrenophora tritici-repentis] & $2 \mathrm{E}-58$ & Ns \\
\hline
\end{tabular}

* Fisher's exact p-value for the comparative analysis on transcript abundance between the mycelial culture (OCL01) and LPPE (OCL03) libraries. 


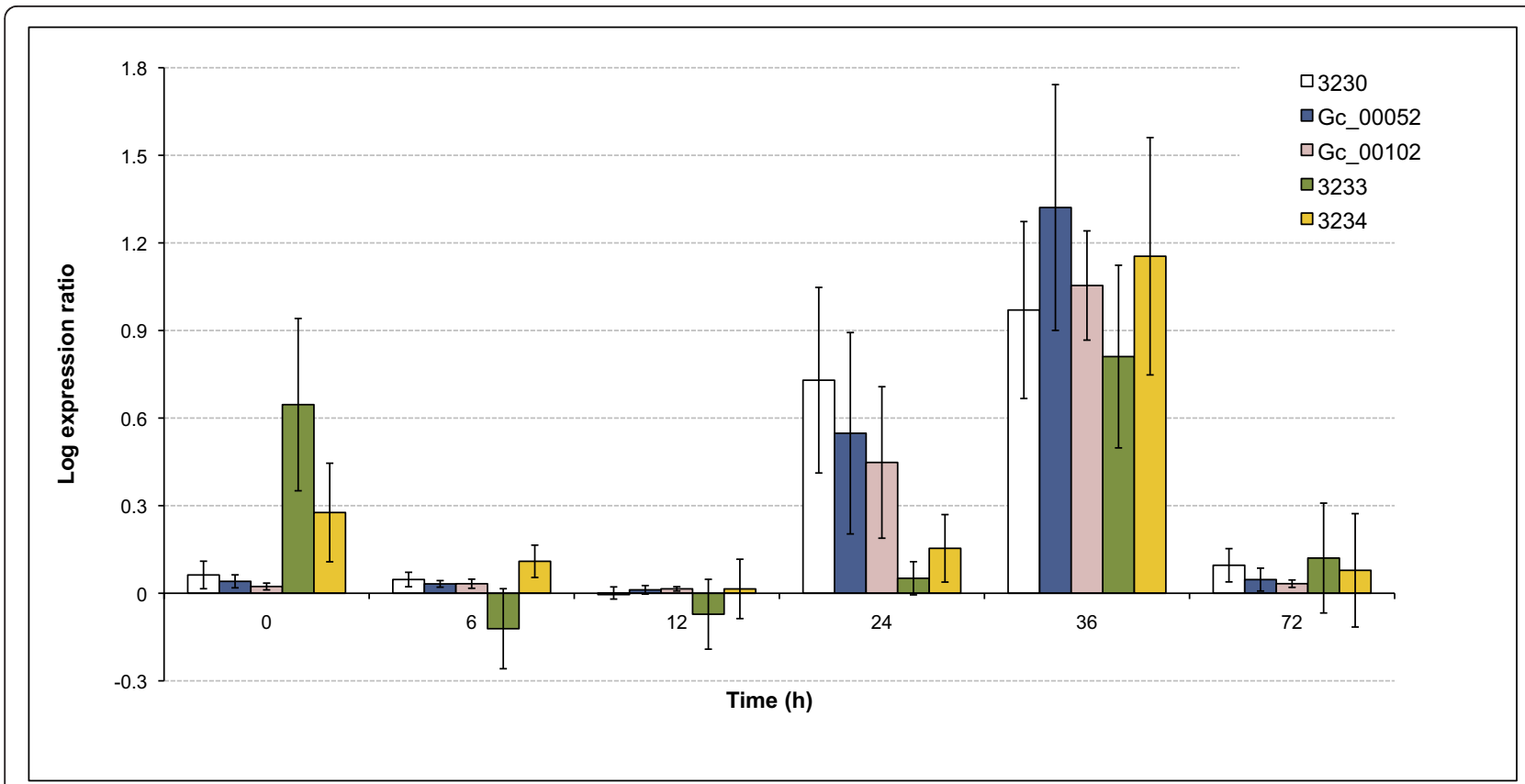

Figure 4 qRT-PCR results showing the expression levels of five unigene-locations at six time points after LPPE treatment relative to their expression levels in cultures of G. clavigera treated with a methanol control solution. (3230 - MFS-transporter, Gc_00052 - P450, Gc_00102 - P450 reductase, 3233 - steroid monooxygenase, 3234 - beta-lactamase)

6,467 ULs. In a separate analysis of the G. clavigera genome sequence we estimated $\sim 9,000$ genes (unpublished results). Based on this number the unigene set represents $\sim 70 \%$ of the G. clavigera genes. Unigene annotation showed that our dataset covers in depth major metabolic pathways for general and essential processes, and provides sequence information on genes that may be unique to G. clavigera. These data will be a useful resource for future research on this pathogen, in particular for its symbiotic association with mountain pine beetle and interaction with the chemical defenses of host trees.

Antisense transcripts may be involved in controlling gene expression $[27,28]$. We estimated that $~ 19 \%$ of $G$. clavigera ULs overlap with another UL from the opposite genome strand, and so potentially represent antisense transcripts. Our analysis indicated, that in most cases contigs containing forward and reverse assembled reads despite unidirectional read orientation (FR contigs) were assemblies of transcripts from overlapping genes. Therefore, FR-filtering can help identify assembly problems as well as potential antisense transcripts in de novo assembled unigene collections.

Control of spore germination may be an important factor in G. clavigera bark beetle symbiosis and tree pathogenicity. Transcript analysis of the spore library indicated that early germination processes may have been induced in our spore samples. We identified several protein chaperones, including two HSP70s (ULs $355,2425)$ and one cyclophillin D (UL 3423) that showed significant similarity to the $A$. nidulans proteins XP_663614, XP_662733, and XP_662187, respectively. Proteome analyses revealed that these proteins accumulated in A. nidulans within 30 to 60 minutes after conidia germination [29]. Other ULs with high transcript frequencies in spores of $G$. clavigera encoded proteins

Table 6 Unigene-locations of cluster 2, which is potentially involved in response to lodgepole pine phloem extract

\begin{tabular}{clcc}
\hline Unigene-location & \multicolumn{1}{c}{ Best annotated protein match from the NCBI nonredundant protein database } & e-value & p-value* \\
\hline 3999 & X-Pro dipeptidyl-peptidase (S15 family) protein [Neosartorya fischeri] & $8 \mathrm{E}-113$ & $\mathrm{~ns}$ \\
4000 & predicted protein [Sclerotinia sclerotiorum] & $1 \mathrm{E}-52$ & $\mathrm{~ns}$ \\
4001 & aromatic ring-opening dioxygenase family protein [Talaromyces stipitatus] & $7 \mathrm{E}-81$ & $\mathrm{~ns}$ \\
4002 & PutA family dehydrogenase [Talaromyces stipitatus] & $2 \mathrm{E}-13$ & $\mathrm{~ns}$ \\
4003 & cupin domain protein [Neosartorya fischeri] & $3 \mathrm{E}-50$ & 0.03 \\
4004 & short chain dehydrogenase [Aspergillus fumigatus] & $4 \mathrm{E}-44$ & $\mathrm{~ns}$ \\
\hline
\end{tabular}

\footnotetext{
* Fisher's exact p-value for the comparative analysis on transcript abundance between the mycelial culture (OCL01) and LPPE (OCL03) libraries.
} 
involved in energy metabolism and protein biosynthesis, which is consistent with respiration and protein biosynthesis activated early in germination [30]. The genes identified in this analysis may serve as markers for early spore germination. However, analysis of gene expression in resting and germinating G. clavigera spores requires further work.

Genes whose transcripts were over-represented in response to LPPE treatment suggested a fungal oxidative stress response caused by host phenolic or other defense compounds present in the phloem extract $[31,32]$. For example, we observed a large number of ESTs for $\mathrm{Cu}$ / $\mathrm{Zn}$ superoxide dismutase (SOD) in the LPPE treatment library (UL 1906). SOD catalyses the conversion of superoxide radicals to molecular oxygen. SOD1 mutants of $N$. crassa are sensitive to superoxide-generating compounds and have a high rate of spontaneous mutations [33]. In contrast, deleting SOD1 did not change the sensitivity of Claviceps purpurea to paraquat and did not affect its pathogenicity [34], indicating that other detoxifying systems may be involved. Interestingly, our EST data revealed a second $\mathrm{Cu} / \mathrm{Zn}$ superoxide dismutase (UL 3288), which does not appear to be upregulated by LPPE treatment, suggesting that the two SODs may have different functions. Similarly, a peroxidase (UL 588) overexpressed in the LPPE treatment library may participate in scavenging reactive oxygen species. Alternatively, it may be involved in detoxification of phenolic compounds, as previous studies have shown that peroxidases can oxidize phenolic substrates and cleave aromatic ring structures $[20,35]$. EST frequencies in the LPPE treatment library were also high for a nitroreductase (UL 4803), but functions of this gene in host colonization are uncertain. Bacterial nitroreductases can detoxify nitrosubstituted compounds [36], including nitrophenols [37] in reactions that produce superoxides and induce oxidative stress [38,39]. In S. cerevisiae two nitroreductases have been identified [40], one of which appears to act in the lipid-signaling pathway. Mutants for these enzymes showed extreme sensitivity to nitrosative substances and have reduced superoxide dismutase activity [41]. The authors hypothesized that the nitroreductases may modulate antioxidant enzymatic activities in yeast.

In filamentous fungi, functionally related genes and genes involved in niche adaptation can occur in clusters. For example, secondary metabolite genes in $A$. fumigatus [42] and genes encoding secreted proteins in Ustilago maydis [43] are clustered. For G. clavigera, we identified two clusters of putatively functionally related genes that were upregulated after LPPE treatment. The first cluster contained five genes: a steroid monooxygenase, a P450, a P450 reductase, a beta lactamase, and an MFS transporter. The steroid monooxygenase and the P450 may participate in detoxification of LPPE compounds by adding or altering functions of metabolites, similar for example to the P450s involved in fungal detoxification of pisatin [44]. The conserved colocalization of the $\mathrm{P} 450$ and the $\mathrm{P} 450$ reductase has been noted in Aspergillus species [45] and led to the hypothesis that this reductase may be specific for the co-localized P450. The observed co-regulation of these two G. clavigera genes in response to LPPE treatment is consistent with this hypothesis. Whether the gene showing high similarity to beta lactamase is involved in ring cleavage of any LPPE metabolite remains to be tested in future work. The second cluster contained an extradiol ring-cleavage dioxygenase and two oxidoreductases, and may be involved in degrading aromatic compounds such as phenolics. Further analyses on genes in these two clusters are necessary to confirm functions and relationships.

\section{Methods}

\section{Fungal isolates and culture conditions}

The eight fungal isolates used in this study are listed in Table 1. To induce a broad spectrum of genes, cultures from each isolate were grown on five different media (Table 2) that varied in nitrogen and carbon sources. For this, we first obtained young mycelial cultures by inoculating plates containing $1 \%$ malt extract agar (MEA, Oxoid, England) overlaid with cellophane with a spore suspension (12 plates/isolate, $5 \times 10^{5}$ spores/ plate), and incubating them at ambient conditions for $48 \mathrm{~h}$. Then, we transferred the cellophane with the young mycelia to new plates that contained the different media (2 plates/isolate/medium). The cultures were grown for four days, and then harvested for RNA extraction, pooling the mycelia from the two plates of each isolate/medium combination.

To identify genes expressed in mycelial culture in response to lodgepole pine phloem extract (LPPE) we grew fungal cultures from the eight isolates on organic nitrogen media (as described above) for $48 \mathrm{~h}$, sprayed them with $200 \mu$ l of crude LPPE, and harvested the mycelia for RNA extraction $48 \mathrm{~h}$ after initial exposure to LPPE. LPPE was prepared as follows: a lodgepole pine bolt from a freshly cut tree was frozen at $-20^{\circ} \mathrm{C}$ and cut into disks. The frozen phloem was separated and ground in a mill with liquid nitrogen. The powder was extracted in 80:20 methanol:water $(2.5 \mathrm{ml} / \mathrm{g})$ and sonicated at $4^{\circ} \mathrm{C}$ for $2 \mathrm{~h}$. After centrifugation, the supernatant was removed and concentrated by $1 / 3$ under a gentle flow of nitrogen gas (final concentration $~ 50: 50$, $\left.\mathrm{MeOH}: \mathrm{H}_{2} \mathrm{O}\right)$. This concentrated crude extract was stored at $-20^{\circ} \mathrm{C}$. To ensure uniform treatment of all cultures, a single preparation of LPPE was used for the experiment. 
For the spore library, cultures of the reference isolate SLKW1407 were grown for seven days on 1\% MEA. Spores were harvested in $5 \mathrm{ml}$ water and filtered through a BD Falcon Cell strainer $(40 \mu \mathrm{m})$ to remove mycelial debris from the spore collection. The spore suspension was centrifuged at 5,000 rpm for $20 \mathrm{~min}$, the pellets were transferred to $1.5 \mathrm{ml}$ microtubes and centrifuged again for $2 \mathrm{~min}$ at 14,000 rpm. These tubes were stored at $-80^{\circ} \mathrm{C}$ until RNA extraction.

\section{RNA isolation, cDNA library construction, cDNA sequencing}

Total RNA was isolated separately from each of the 48 isolate/media/LPPE treatment variants and the spores, respectively, using the RNeasy Mini Plant RNA isolation kit (Qiagen, Mississauga, ON, Canada). The total RNA samples were then treated with DNaseI (Fisher Scientific, Ottawa, ON, Canada), analyzed for quality by spectrophotometer and agarose gel analysis, and quantified. To generate comparable treatments for the reference isolate, and to ensure high sequencing efficiency, we pooled equal amounts of total RNA from the different isolate/media/LPPE treatment variants and prepared four isolate/treatment combinations: three treatments with the reference isolate, and one pool containing total RNA from the other seven isolates (Table 3). From the resulting four RNA samples we purified poly $(\mathrm{A}+)$ mRNA using the Oligotex mRNA purification kit (Qiagen), and generated first strand cDNA using SuperScript III reverse transcriptase (Invitrogen, Carlsbad, CA, USA), CDS-3M primer (Evrogen, Moscow, Russia), and SMART IV Oligonucleotide (Clontech, Mountain View, CA, USA). Second strand cDNA was prepared by long distance-PCR with Phusion Hot Start DNA Polymerase (Finnzymes, Espoo, Finland). The cDNA samples were split into two fractions and one fraction was normalized using the TRIMMER-DIRECT cDNA normalization kit (Evrogen). For library construction, normalized and non-normalized cDNA was digested with SfiI and size fractionated. The fractions $>500 \mathrm{bp}$ were directionally cloned into the SfiI-digested pDNR-LIB vector (Clontech). A set of 25,000 clones randomly selected from all libraries were partially sequenced on a 3730XL DNA analyzer (Applied Biosystems, Carlsbad, CA, USA) from the 5' and 3' ends using the -21 M13 forward and M13 reverse primers, respectively. Sequencing was done at the British Columbia Cancer Agency Genome Sciences Centre (Vancouver, BC, Canada).

\section{qRT-PCR analysis}

For quantitative real time PCR (qRT-PCR) we grew the reference isolate SLKW1407 on organic nitrogen media (as described above) and treated cultures with either LPPE, or a methanol control solution $(50: 50, \mathrm{MeOH}$ :
$\mathrm{H}_{2} \mathrm{O}$ ). We harvested mycelia at $0,6,12,24,36,48$, and $72 \mathrm{~h}$ after the beginning of treatment, and proceeded with qRT-PCR analysis following the protocol described by DiGuistini et al. [13]. For each time point we prepared three biological and three technical replicates. The primers used to amplify the transcript of interest are shown in Table 7. Data collection and statistical analysis were conducted with the Roche CFX 96-real-time PCR detection system (Roche, Quebec, CA).

\section{EST assembly}

We processed chromatograms and trimmed low quality sequences using PHRED [46], requiring a minimum of 100 bp with quality scores above PHRED 20. Using cross-match (http://www.phrap.org/), we removed vector sequences and filtered the remaining sequences for Escherichia coli. To account for possible contamination from the lab environment we also screened for Saccharomyces cerevisiae, Agrobacterium, and Aspergillus spp. To the resulting set of 44,288 quality-filtered reads we added 5,950 quality-filtered G. clavigera reads (isolate SLKW1407) from previous work [13]. Since the cDNA libraries contained directionally cloned inserts, sequences obtained using the M13 reverse primer (3' reads) had a 3' -5 ' orientation with respect to the original mRNA. These 3' reads were reverse-complemented to produce a 5'-3' oriented read collection. Then we removed the polyA tails, which interfere in contig assembly, and discarded sequences with long mononucleotide stretches. The resulting set of 50,167 high-quality reads was assembled with CAP3 [47] using default settings except for a minimum overlap of $40 \mathrm{bp}$ and a minimum identity of $95 \%$.

Because the reads were 5'-3' oriented prior to assembly, most of the unigenes had a 5'-3' orientation. This was verified by comparing the input fasta files and the assembler's ACE-format output files. Then, we screened the ACE file for contigs that contained reads in both orientations. Because proteins are encoded on the

$\begin{aligned} & \text { Table } 7 \text { Primers used for quantitative RT-PCR analysis of } \\
& \text { unigene locations (ULs) }\end{aligned}$
\begin{tabular}{lll}
\hline Target UL & Primer name & $\mathbf{5}^{\prime}-\mathbf{3}^{\prime}$ sequence \\
\hline 3230 & R1718-F1 & GTG TCC TCC ACC TTC CTC ACC \\
& R1718-R1 & CGT GAC TCC CTT GAC TTC TGG G \\
GC_00052 & GC_0052-F1 & GCT CTC TCT TाT GCC GGC GGA \\
& GC_0052-R1 & GAG CCG GCC AGC GT GAG TAA \\
GC_00102 & GC_00102-F1 & TCG GAC GGA CTG CAA ACG CG \\
& GC_00102-R1 & CGA GCC CCA GAA AAG GAC GAC \\
3233 & R1719-F1 & CTC AGC AAC GGT CCA ACC TC \\
& R1719-R1 & GTG CTT CTT CCA CTT GCG GG \\
3234 & F1718-F3 & GAGCTGCTGACGCTCGATA \\
& F1718-R3 & ACCTGACTGCTGTCGTCCAT
\end{tabular}


forward strand of the transcript, such contigs potentially represent misassembled unigenes.

\section{Unigene-locations on reference genome}

The unigenes were mapped against the G. clavigera genome [24] using BLAT [48]. For each unigene, we selected the best hit to the genome, and recorded sequence similarity (\%) and unigene alignment length (\%). Since all unigenes were 5'-3' oriented they mapped to the genome strand that encoded the respective gene. To group unigenes that represented the same gene, we generated unigene-locations (ULs) based on strand-specific overlap of unigenes on the genome, and linking information from unassembled 5'-3' clone pairs. We verified unigene assembly by mapping all reads to the genome with BLAT and testing whether the unassembled reads of a contig mapped to the same genome location as the contig.

\section{Unigene annotation}

For annotation, all unigenes were compared to the non-redundant NCBI protein database using BLAST (NCBI, Bethesda, MD, USA; min e-value $1.0 \times 10^{-4}$ ). We used custom Perl scripts to capture the best BLAST hit on the forward frame and determine the most likely open reading frame (ORF) for each sequence. For the latter, all ORFs longer than 25 amino acids were identified using CLC Genomics Workbench (CLC bio, Aarhus, Denmark). If the sequence had a significant BLAST match on the forward frame, the best ORF covering that match and the most likely transcription start and stop were determined, keeping track of $5^{\prime}$ and 3' ORF truncations. If no BLAST match was available, the longest ORF on the forward frame was selected for the sequence. In addition, unigenes were annotated with InterPro IDs and Gene Ontology terms using InterProScan (EBI, Cambridge, UK), and assigned K-numbers with corresponding KEGG-BRITE classifications using KAAS [49]. To evaluate unigene representation in essential metabolic pathways, we conducted a reciprocal BLAST analysis on the translated G. clavigera unigene ORFs against the annotated Magnaporthe grisea and Aspergillus fumigatus protein datasets downloaded from the Broad Institute (http://www.broadinstitute.org/).

\section{Statistical analysis}

We identified potentially differentially expressed genes by comparing read frequencies in the non-normalized, reference isolate cDNA libraries constructed from (i) mycelial cultures, (ii) cultures treated with LPPE, and (iii) spores. To account for unigene redundancy per gene we assessed library-specific read frequencies per UL rather than per unigene. Also, we counted only one read per transcript, and ignored reverse-assembled reads in contigs. After normalizing the read counts, we performed pair wise comparisons of libraries using a modified Fisher's exact test.

\section{Additional material}

Additional file 1: Table S1. Excel file containing the supplementary table with all unigene locations, their coordinates on the G. clavigera genome, the ESTs that mapped to each UL, relevant annotations, and expression analysis results.

\section{Acknowledgements}

We thank Thomas Wang for technical assistance with qRT-PCR and Greg Taylor for reviewing the technical part of the manuscript. This work was supported with grants from the Natural Sciences and Engineering Research Council of Canada (to JB and CB), and with funds from Genome Canada, Genome British Columbia, and Genome Alberta in support of the Tria 1 and Tria 2 projects (http://www.thetriaproject.ca; to JB, CB, CIK, and SJMJ). Salary support for JB was provided, in part, by the UBC Distinguished University Scholar program.

\section{Author details}

${ }^{1}$ Department of Wood Science, University of British Columbia, Vancouver, Canada. ${ }^{2}$ Michael Smith Laboratories, University of British Columbia, Vancouver, Canada. ${ }^{3}$ BC Cancer Agency Genome Sciences Centre, Vancouver, Canada.

\section{Authors' contributions}

UH performed the data analysis and drafted the manuscript. SD designed and performed experiments with the fungi and prepared the MRNA. CIK, ML and $\mathrm{HH}$ constructed the CDNA libraries. YW performed the GRT-PCR. TRD and NYL conducted the EST assembly. RAH directed EST sequencing. GR participated in drafting the technical parts of the manuscript. SJMJ, CB and JB conceived and directed the project. All co-authors critically reviewed and edited the manuscript. All authors read and approved the final manuscript.

Received: 8 May 2010 Accepted: 4 October 2010

Published: 4 October 2010

\section{References}

1. Reid RW, Whitney HS, Watson JA: Reactions of lodgepole pine to attack by Dendroctonus ponderosae Hopkins and blue stain fungi. Can J Bot 1967, 45:1115-1126.

2. Zipfel RD, de Beer ZW, Jacobs K, Wingfield BD, Wingfield MJ: Multi-gene phylogenies define Ceratocystiopsis and Grosmannia distinct from Ophiostoma. Stud Mycol 2006, 55:75-97.

3. Yamaoka Y, Hiratsuka Y, Maruyama PJ: The ability of Ophiostoma clavigerum to kill mature lodgepole pine trees. Eur J For Pathol 1995, 25:401-404.

4. Lee S, Kim JJ, Breuil C: Pathogenicity of Leptographium longiclavatum associated with Dendroctonus ponderosae to Pinus contorta. Can J For Res 2006, 36:2864-2872.

5. Six DL: A comparison of mycangial and phoretic fungi of individual mountain pine beetles. Can J For Res 2003, 33:1331-1334.

6. Paine TD, Raffa KF, Harrington TC: Interactions among scolytid bark beetles, their associated fungi and live host conifers. Annu Rev Entomol 1997, 42:179-206.

7. Krokene $P$, Nagy NE, Krekling T: Traumatic resin ducts and polyphenolic parenchyma cells in conifers. In Induced plant resistance to herbivory. Edited by: Schaller A. Springer, Dordrecht; 2008:147-169.

8. Keeling $\mathrm{Cl}$, Bohlmann J: Genes, enzymes and chemicals of terpenoid diversity in the constitutive and induced defense of conifers against insects and pathogens. New Phytol 2006, 170:657-675.

9. Keeling $\mathrm{Cl}$, Bohlmann J: Diterpene resin acids in conifers. Phytochem 2006, 67:2415-2423. 
10. Blanchette RA, Farrell RL, Burnes TA, Wendler PA, Zimmerman W, Brush TS, Snyder RA: Biological control of pitch in pulp and paper production by Ophiostoma piliferum. TAPPI J 1992, 75:102-106.

11. Brush TS, Farrell RL, Ho C: Biodetoxification of wood extractives from southern yellow pine by Ophiostoma piliferum. TAPPI J 1994, 77:155-159.

12. Wang Z, Chen T, Gao Y, Breuil C, Hiratsuka Y: Biological degradation of resin acids in wood chips by wood-inhabiting fungi. Appl Environ Microbiol 1995, 61:222-225.

13. DiGuistini S, Ralph SG, Lim YW, Holt R, Jones S, Bohlmann J, Breuil C: Generation and annotation of lodgepole pine and oleoresin-induced expressed sequences from the blue-stain fungus Ophiostoma clavigerum, a mountain pine beetle-associated pathogen. FEMS Microbiol Lett 2007, 267:151-158.

14. Hergert HL: The flavonoids of lodgepole pine bark. J Org Chem 1956, 21:534-53.

15. Vercruysse SAR, Delcour JA, Dondeyne P: Isolation of quercetin, myricetin, and their respective dihydro-compounds by Sephadex $\mathrm{LH}-20$ chromatography. J Chrom 1985, 324:495-497.

16. Matthews S, Mila I, Scalbert A, Pollet B, Lapierre C, Herve' du Penhoat CLM, Rolando C, Donnelly DMXL: Method for estimation of proanthocyanidins based on their acid depolymerization in the presence of nucleophiles. J Agric Food Chem 1997, 45:1195-1201.

17. Stolter C, Niemela P, Ball JP, Julkunen-Tiitto R, Vanhatalo A, Danell K, Varvikko T, Ganzhorn JU: Comparison of plant secondary metabolites and digestibility of three different boreal coniferous trees. Basic Appl Ecol 2009, 10:19-26.

18. Witzell J, Martin JA: Phenolic metabolites in the resistance of northern forest trees to pathogens - past experiences and future prospects. Can J For Res 2008, 38:2711-2727.

19. Kocabas DS, Bakir U, Phillips SE, Mcpherson MJ, Ogel ZB: Purification, characterization and identification of a novel bifunctional catalasephenol oxidase from Scytalidium thermophilum. New Biotechnol 2009, 25 S92-93.

20. Band L, Ciofi-Baffoni S, Tien M: Lignin and Mn-peroxidase-catalyzed oxidation of phenolic lignin oligomers. Biochem 1999, 38:3205-3210.

21. Hammer E, Schoefer L, Schäfer A, Hundt K, Schauer F: Formation of glucoside conjugates during biotransformation of dibenzofuran by penicillium canescens SBUG-M 1139. Appl Microbiol Biotechnol 2001, 57:390-394.

22. McCue $P$, Shetty $K$ : Role of carbohydrate-cleaving enzymes in antioxidant mobilization from whole soybean fermented with Rhizopus oligosporus. Food Biotechnol 2003, 17:27-37.

23. Nicholson RL, Hipskind J, Hanau RM: Protection against phenol toxicity by the spore mucilage of Colletotrichum graminicola, an aid to secondary spread. Physiol Mol Plant Pathol 1989, 35:243-252.

24. DiGuistini S, Liao NY, Platt D, Robertson G, Seidel M, Chan SK, Birol I, Holt RA, Hirst M, Mardis E, Marra MA, Hamelin RC, Bohlmann J, Breuil C, Jones SJM: De novo genome sequence assembly of a filamentous fungus using Sanger, 454 and Illumina sequence data. Genome Biol 2009, 10:R94.

25. Nagaraj SH, Deshpande N, Gasser RB, Ranganathan S: ESTExplorer: an expressed sequence tag (EST) assembly and annotation platform. Nucleic Acids Res 2007, 35:W143-147.

26. Tang Z, Choi JH, Hemmerich C, Sarangi A, Colbourne JK, Dong Q: ESTPiper - a web-based analysis pipeline for expressed sequence tags. BMC Genomics 2009, 10:174

27. Makalowska I, Lin CF, Makalowski W: Overlapping genes in vertebrate genomes. Comput Biol Chem 2005, 29:1-12.

28. Smith CA, Robertson D, Yates B, Nielsen DM, Brown D, Dean RA, Payne GA: The effect of temperature on Natural Antisense Transcript (NAT) expression in Aspergillus flavus. Curr Genet 2008, 54:241-69.

29. Oh YT, Ahna CS, Kima JG, Roa HS, Lee CW, Kim JW: Proteomic analysis of early phase of conidia germination in Aspergillus nidulans. Fungal Genet Biol 2010, 47:246-253.

30. Taubitz A, Bauer B, Heesemann J, Ebel F: Role of respiration in the germination process of the pathogenic mold Aspergillus fumigatus. Curr Microbiol 2007, 54:354-360.

31. Takatsume $Y$, Maeta K, Izawa S, Inoue Y: Enrichment of yeast thioredoxin by green tea extract through activation of Yap1 transcription factor in Saccharomyces cerevisiae. J Agric Food Chem 2005, 53:332-337.
32. Kim JH, Campbell BC, Mahoney N, Chan KL, May GS: Targeting antioxidative signal transduction and stress response system: control of pathogenic Aspergillus with phenolics that inhibit mitochondrial function. J Appl Microbiol 2005, 101:181-189.

33. Chary P, Dillon D, Schroeder AL, Natvig DO: Superoxide dismutase (sod-1) null mutants of Neurospora crassa: oxidative stress sensitivity, spontaneous mutation rate and response to mutagens. Genetics 1994 137:723-730

34. Moore S, De Vries OMH, Tudzynski P: The major Cu,Zn SOD of the phytopathogen Claviceps purpurea is not essential for pathogenicity. Molec Plant Pathol 2002, 3:9-22

35. Xia Z, Yoshida T, Funaoka M: Enzymatic degradation of highly phenolic lignin-based polymers (lignophenols). Eur Polym J 2003, 39:909-914.

36. Bryant C, McElroy WD: Nitroreductases. In Chemistry and Biochemistry of Flavoenzymes. Volume II. Edited by: Muller F. CRC Press: Boca Raton, FL; 1991:291-304.

37. Dai R, Chen J, Lin J, Xiao S, Chen S, Deng Y: Reduction of nitro phenols using nitroreductase from $E$. coli in the presence of $\mathrm{NADH}$. J Hazard Mater 2009, 170:141-143.

38. Peterson FJ, Mason RP, Hovespian J, Holtzman JL: Oxygen-sensitive and -insensitive nitroreduction by Escherichia coli and rat hepatic microsomes. J Biol Chem 1979, 254:4009-4014

39. Angermaier $\mathrm{L}$, Simon $\mathrm{H}$ : On nitroaryl reductase activities in several clostridia. H-S Z Physiol Chem 1983, 364:1653-1663.

40. de Oliveira IM, Henriques JA, Bonatto D: In silico identification of a new group of specific bacterial and fungal nitroreductases-like proteins. Biochem Biophys Res Commun 2007, 355:919-925.

41. de Oliveira IM, Zanotto-Filho A, Fonseca Moreira JC, Bonatto D, Pegas Henriques JA: The role of two putative nitroreductases, Frm2p and $\mathrm{Hbn} 1 \mathrm{p}$, in the oxidative stress response in Saccharomyces cerevisia. Yeast 2010, 27:89-102

42. Nierman WC, Pain A, Anderson MJ, Wortman JR, Kim HS, Arroyo J, Berriman B, Abe K, Archer DB, Bermejo C, et al: Genomic sequence of the pathogenic and allergenic filamentous fungus Aspergillus fumigatus.. Nature 2005, 438:1151-1156.

43. Kämper J, Kahmann R, Bölker M, Ma LJ, Brefort T, Saville BJ, Banuett F, Kronstad JW, Gold SE, Müller O, et al: Insights from the genome of the biotrophic fungal plant pathogen Ustilago maydis. Nature 2006 444:97-101.

44. Matthews DE, VanEtten HD: Detoxification of the phytoalexin pisatin by a fungal cytochrome P-450. Arch Biochem Biophys 1983, 224:494-505.

45. Kelly DE, Krasevec N, Mullins J, Nelson DR: The CYPome (Cytochrome P450 complement) of Aspergillus nidulans. Fungal Genet Biol 2009, 46:S53-61.

46. Ewing B, Green P: Base-calling of automated sequencer traces using phred. II. Error probabilities. Genome Res 1998, 8:186-194.

47. Huang X, Madan A: CAP3: a DNA sequence assembly program. Genome Res 1999, 9:868-877.

48. Kent WJ: BLAT-the BLAST-like alignment tool. Genome Res 2002, 12:656-664.

49. Moriya Y, Itoh M, Okuda S, Yoshizawa A, Kanehisa M: KAAS: an automatic genome annotation and pathway reconstruction server. Nucleic Acids Res 2007, 35:W182-185.

\section{doi:10.1186/1471-2164-11-536}

Cite this article as: Hesse-Orce et al:: Gene discovery for the bark beetlevectored fungal tree pathogen Grosmannia clavigera. BMC Genomics 2010 11:536. 